\title{
EL VALOR DE LA INFORMACION DESDE LA \\ PERSPECTIVA DEL USUARIO Y EL PAPEL DE LAS \\ BIBLIOTECAS $Y$ UNIDADES DE INFORMACION EN LA EMPRESA
}

\author{
Belén Altuna Esteibar*
}

Resumen: Se presentan distintas aproximaciones al concepto de "valor de la información" desde la perspectiva del usuario final, analizando a continuación los flujos de información y el papel de las fuentes formales de información en el contexto del proceso tecnológico industrial. Finalmente, se sugiere la importancia para el bibliotecario/especialista en información del uso de las características de un entorno dado con el fin de optimizar el valor potencial de la información.

Palabras clave: Valor de la información, servicios de información en la empresa, patrones de uso de información, flujos de comunicación.

\begin{abstract}
Some approaches to the concept of «information value» from the user perspective are reviewed. Then, focusing on the context of the technological innovation process within corporations, the flow of information and the role of formal information sources in that process as well are studied. Finally, the relevance for the librarian/information specialist of the use of a given environment characteristics in order to optimize the potential value of information is suggested.

Keywords: Information value, information services in corporations, information use patterns, communication flow.
\end{abstract}

\section{Introducción}

Parafraseando una expresión popular en sectores económicos y políticos, se podría decir que la industria de la información goza de "buena salud» en España. Aunque la tendencia en cuanto a producción de bases de datos sigue estando netamente en el ámbito de los organismos públicos, que representan un $71,76 \%$ del total, en los últimos tres años el parque de oferta de servicios de información electrónica ASCII ha aumentado a 216, lo que supone un incremento de un $30,09 \%$ respecto a $1988(1)$.

Las cifras sugieren la pregunta por la información en la industria española. Nuestro país, como el resto de los países menos desarrollados de la Comunidad Económica Europea, posee una estructura empresarial fundamentalmente basada en pequeñas y medianas empresas cuyos hábitos de adquisición de información han consistido, y consisten todavía en muchos casos, en las fuentes tradicionales, como son los distribuidores de productos, los clientes, algunas revistas de divulgación, etc.

* Biblioteca Nacional. Madrid. Recibido 18-3-92. 
Sin embargo, con la creación del Mercado Unico Europeo, a la vez que se está estimulando la actividad en el mercado empresarial y comenzando a cambiar la fisonomía industrial del país, se empieza a considerar la información como un elemento esencial para poder competir y sobrevivir al vecino europeo.

A la vista de ello, se podría pronosticar un incremento progresivo en la demanda de servicios de información por parte de la industria, e igualmente, en algunos casos, la creación de centros de información en las propias industrias, que atenderían unas clientelas especializadas como son los gestores de empresa, los departamentos de marketing, de investigación y desarrollo $(I+D)$, etc. Las necesidades de estos colectivos están bien diferenciadas, así como el modo de provisión de información.

Esto nos lleva a plantearnos el papel de la información en el desarrollo industrial, el valor añadido que el aprovisionamiento de información puede suponer en el éxito tecnológico y empresarial, el cómo pensar el centro de información en un ámbito cuyas premisas son puramente económicas en el cual lo que existe, existe en la medida en que "produce» o "crea" beneficios en este entorno económico dado.

Como señalan Cronin y Gudim (2), la pregunta más comúnmente formulada en la literatura para acercarse al valor de la información es: información para quién y, en esa medida, se contempla al usuario como variable esencial así como los beneficios generados por el uso de la información, en lugar de centrarse en el sistema de información en sí mismo.

Este artículo dará cuenta someramente de algunas de las aproximaciones teóricas al valor de la información desde la perspectiva mencionada, analizando después en un contexto específico, el del proceso de innovación tecnológica industrial, el flujo de información y el papel de las fuentes formales de información en dicho proceso. Finalmente, se sugiere el uso que el bibliotecario o especialista en información podría hacer de las características de un contexto dado con el fin de optimizar el valor potencial de la información.

\section{Enfoques sobre el valor de la información desde la perspectiva del usuario}

La definición de "valor de la información» desde la perspectiva del usuario presume, como señala Taylor (3) que un mensaje no tiene valor inherente sino que adquiere valor en un contexto determinado siendo el usuario quien lo asigna. Para Taylor, la utilización de la información no depende tan solo de la facilidad de acceso, de su calidad y validez, variables usadas muy frecuentemente para explicar el valor de la información, sino también del grado de correspondencia entre el conocimiento adquirido por la información y el medio en el que el usuario opera y donde debe tomar decisiones. La implicación de este presupuesto es, pues, que la información tiene valor potencial pero sólo en un contexto determinado en que el usuario determinará su valor a través de su utilidad, se podrá hablar en términos de "valor de la información».

Marschak (4) define el "valor de la información» como los beneficios generados por su uso óptimo. Se centra en el hecho de que la utilidad de la información es variable dependiendo de las circunstancias y el factor tiempo de tal manera que información válida en un momento dado puede perder su valor en un momento 
ulterior. Otro partidario del valor de la información referido a un contexto determinado es Ozog (5) quién defiende que el valor de una pieza específica de información es igual a lo que se ganaría en utilidad por optar por una mejor elección entre acciones terminales o definitivas.

La aproximación de King (6) plantea tres vías para juzgar el valor de la información desde el punto de vista del usuario: la "perspectiva de inversión» (input perspective), la "perspectiva del proceso" (process perspective) y la "perspectiva del rendimiento" (output perspective). En la primera, se juzga el valor de la información por el precio que el usuario estaría dispuesto a pagar por los servicios de información. La perspectiva del proceso define el valor de la información por el modo en que el uso de la información afecta a la actividad o medio donde se utiliza. Por último, la perspectiva del rendimiento consiste en determinar el valor en función de la utilidad de la información para la consecución de objetivos previamente establecidos.

Repo (7) propone lo que él llama una «aproximación dual al valor de la información" (dual approach to the value of information). Distingue entre lo que serían valores prácticos o instrumentales de la información y aquéllos de carácter intrínseco o filosófico. Considerando los valores prácticos para nuestro propósito, éstos se subdividirian en valor de uso y valor de cambio, siendo el primero definido como el beneficio obtenido por el usuario mediante el uso de la información y el consecuente efecto de tal uso. Por el contrario, el valor de cambio estaría relacionado con el valor de productos de información en el mercado.

Aunque Repo analiza en mayor profundidad diferentes tipos de valor de uso, lo que nos interesa aquí subrayar es la consideración del valor de uso no sólo en función de la accesibilidad, validez, etc., que son obviamente componentes necesarias sino, además, en función del grado de adecuación entre la provisión de información y el contexto o medio en el que el usuario actúa. De alguna manera es similar al enfoque de Taylor (3) en tanto que Repo también define el valor de la información asociado a un usuario en un contexto determinado.

Algunos autores como Repo (7) subrayan que no tiene sentido el tratar de calcular el valor total de la información y, aunque otros son más moderados en sus postulados, existe un consenso casi general en las dificultades de asignar valor a la información. No obstante, a la vista del breve resumen presentado anteriormente, parece innegable la consideración del usuario y el contexto en el que éste actúa a la hora de analizar el valor de la información.

Ahora bien, desde nuestro punto de vista, el medio o contexto en el que el individuo utiliza información es, a su vez, «informacionalmente» complejo, esto es, existen múltiples canales y fuentes de información interactuando en dicho medio. Se sugiere aquí que, para conseguir el máximo valor de la información desde la perspectiva del usuario, habria que tener en cuenta también la estructura del flujo de información en cada medio específico. De igual modo, en la medida en que los bibliotecarios/especialistas en información somos proveedores de bienes informativos, deberíamos tomar en consideración no sólo las necesidades de información del individuo aisladamente, sino en conjunción con el complejo y diverso sistema de flujos de información de su medio, en la medida en que nuestros objetivos profesionales estriban en la creación de servicios que añadan el máximo valor potencial a la información. 
Analicemos las afirmaciones anteriores aplicándolas a un tipo específico de usuario y sus hábitos de información en un contexto particular, resaltando la estructura del flujo informativo.

\section{El flujo de información en la industria}

Hasta el momento hemos identificado las dos variables más importantes para definir el valor de la información del usuario: el uso de la misma y el contexto en que se usa; sigue, sin embargo, siendo una incógnita cómo medir estas variables, cómo asignarles valores. Brevemente, además, sosteníamos la dificultad de aislar una pieza de información y medir su efecto en función de los supuestos beneficios que generaría al usuario, ya que éste se encuentra permanentemente emitiendo y recibiendo información a través de múltiples canales. Por otra parte, el usuario puede tener también un conocimiento y una información previas que aplica directamente cada vez que lo necesita. Así pues, se plantea la hipótesis de que una pieza de información no es más que una parte, una porción integrada en un sistema más amplio en el que interactúan múltiples mensajes y canales de información. Esto daría cuenta de por qué el mismo mensaje o pieza de información adquiere diferente valor para diferentes individuos e, incluso, para el mismo individuo en distintas situaciones.

Para ahondar en la importancia de los flujos de información para la definición de su impacto en la creación de servicios de información centrados en el usuario vamos a analizar un proceso específico como es el de la innovación tecnológica en el ámbito industrial. Se ha elegido este proceso porque requiere de gran volumen de información, se ha investigado en profundidad sobre él y, por tanto, existe abundante evidencia de su complejidad y de su estructura.

Otras razones que nos han llevado a seleccionar el proceso de innovación tecnológica se derivan del hecho de que los éxitos de la actividad tecnológica tienen un reflejo inmediato, un impacto económico cuantificable en el mercado - como diría Repo (7) «en el mundo de los valores de cambio»- y asimismo en el desarrollo y supervivencia de las industrias existentes, al menos en una economia de mercado. De hecho, los patrones de comunicación y los flujos de información han sido ampliamente estudiados, debido a su incidencia en la resolución con éxito de los procesos de innovación tecnológica en equipo y, consecuentemente, a la importancia de la comunicación para la productividad y desarrollo de la organización empresarial.

\section{La innovación tecnologica}

De acuerdo con Utterback (8) un invento es una "solución original que resulta de la sintesis entre la información sobre una necesidad o carencia e información sobre los medios técnicos con los cuales esa necesidad o carencia puede ser cubierta" (an invention is an original solution resulting from the synthesis of information about a need or want and information about the technical means with which the need or want may be met). El autor se refiere a producto innovador como 
un invento que ya se ha introducido en el mercado mientras que proceso innovador sería el primer uso durante el proceso de producción. Utterback considera tres fases solapadas: las dos primeras, generación de la idea y resolución de problemas están relacionadas y concluyen en un invento, tal como se ha definido más arriba, y la tercera corresponde a la fase de implementación que da lugar a una innovación (invención ya en el mercado). Otras definiciones, aunque también incorporan estas tres fases, no distinguen entre invento e innovación.

En el estudio de Battelle Columbus Laboratories (9) se define la innovación tecnológica como «una compleja serie de actividades que comienza cuando la idea original es concebida; prosigue con una sucesión de pasos entretejidos de investigación, desarrollo, ingeniería, diseño, análisis del mercado, toma de decisiones de gestión, etc.; y termina con la primera realización; cuando un producto industrial con éxito, que puede ser un objeto, una técnica, o un proceso, es aceptado en el mercado" (a complex series of activities beginning at first conception when the original idea is conceived; proceeding through a succession of interwoven steps of research, development, engineering, design, market analysis, management decision making, etc.; and ending at first realization; when an industrially successful product which may actually be a thing, a technique, or a process, is accepted in the marketplace). Nótese que las tres fases mencionadas por Utterback (8) están también implícitamente en la definición de Battelle (9).

El proceso de innovación tecnológica ocurre y se desarrolla en un medio, un contexto «informacional» en el que canales informales de información como los contactos y las conversaciones entre personas están interactuando con literatura técnica formal en aras del éxito del proceso. Obviamente, todos estos canales están sostenidos por los individuos, los innovadores en este caso, que son transformadores, sintetizadores, receptores y emisores de mensajes y cuyos patrones de comunicación y características de uso de la información van a jugar un importante papel en el proceso de innovación.

\section{Modelos de uso de información de los innovadores tecnológicos}

Como lo que nos concierne en este trabajo es el valor de la información desde la perspectiva del usuario, antes de fijarnos en las particularidades de los flujos y necesidades de información en cada fase del proceso innovador, vamos a presentar los modelos de uso de la información de los tecnólogos estudiados por Allen (10). El subraya que, aunque la tecnología utiliza ampliamente la información en cada fase del proceso y teniendo los científicos y tecnólogos necesidades similares de información en el proceso creador, sin embargo difieren absolutamente en el producto final. El material primario y el resultado final de la ciencia básica es la información en forma de lenguaje (escrito, matemático, etc.) mientras que la tecnología se abastece de información y produce un proceso o producto que, aun conteniendo información, ésta está codificada en el proceso o producto mismo.

Así pues, según Allen (10), el tecnólogo obtiene información descodificándola y descifrándola del proceso tecnológico $\mathrm{y}$, por tanto, debe confiar más firmemente que los científicos en la comunicación personal con sus colegas. Esto lleva a plantearse el diseño de sistemas apropiados de aprovisionamiento y diseminación 
de información de modo diferente en un caso y en el otro, si los proveedores de información intentan maximizar el valor potencial de sus servicios.

Hay en el estudio de Allen (10) algunos otros modelos de uso de la información que son interesantes de señalar, tales como el menor tiempo que los tecnólogos dedican a la lectura y el menor volumen de publicaciones producidas que los científicos. Por otra parte, los científicos desarrollan fuertes "colegios invisibles» para mantener relaciones con sus colegas y estar actualizados respecto a los avances en sus áreas, mientras que los tecnólogos frecuentemente tienen barreras en las empresas para transmitir información libremente fuera de ellas y tienden a asociarse estrechamente con sus colaboradores dentro de la firma. Para el tecnólogo la información generada internamente es una fuente de información extremadamente importante y no puede ser publicada en los canales de información habituales, pues se considera propiedad de la empresa y, consecuentemente, debe ser protegida.

Todas estas características del medio, la naturaleza de la información tecnológica y los patrones de uso de la información de los tecnólogos configuran y determinan el flujo de información en el proceso innovador.

\section{Fases de la innovación tecnológica y flujos de información}

A pesar de que existe cierto solapamiento en las fases del proceso de innovación tal como son expresadas por Utterback (8), muchos estudios han tratado de aislar particularidades en el uso de la información en cada una de ellas. Parece razonable presumir que las fuentes de información utilizadas al confrontar un problema pudieran diferir de las usadas en la resolución de problemas. Vamos a estudiar aqui las dos primeras fases definidas como generación de ideas y resolución de problemas, ya que la tercera fase, la de implementación, está más ligada a otro tipo de usuarios, que serían los relacionados con marketing, ventas y gestión que, a su vez, tendrán unas necesidades de información distintas, así como diversos serán los usos que de ella harán. Se debe señalar que los resultados de los trabajos de investigación sobre flujos de información no son unánimes, como ocurre casi siempre en los estudios realizados con las metodologías propias de las ciencias sociales. Aun así vamos a revisar los trabajos considerados más relevantes enfatizando aquellos resultados más ampliamente aceptados.

\section{Fase de generación de ideas}

Goldhar, Bragaw y Schwartz (11), resumiendo los resultados habidos en el área de estimulación de ideas en el proceso de innovación, concluyen que entre $2 / 3$ y $3 / 4$ de innovaciones exitosas son estimuladas por información sobre una necesidad de mercado (reconocimiento de una demanda potencial). Utterback (8), yendo un paso más lejos, afirma que la información técnica reciente y actual no parece ser un estímulo para la innovación. Esta conclusión se basa en el análisis de las fuentes de información utilizadas en la fase de generación de ideas.

Los mencionados Goldhar et al. (11) diferencian entre fuentes y canales de información. En sus resultados, el "mayor estímulo" se debe en un $58 \%$ a la 
formación profesional y a la experiencia anterior, mientras que un $8 \%$ es debido al conocimiento actual de los innovadores en su disciplina. Los artículos técnicos y patentes son el mayor estímulo solamente en un $7 \%$ de los casos. Estos resultados coinciden con los de Utterback (8), quien señala que las fuentes principales de información técnica en el desarrollo de la innovación son la experiencia y la preparación profesional.

Entre los canales de información con mayor valor como estímulos, Goldhar et al. (11) en el mismo estudio, hallan que la discusión informal con los colegas de la empresa tiene el mayor impacto para un $45 \%$ de los entrevistados, mientras que el $8 \%$ responden que el mayor impacto es debido a discusión informal con colegas de otras empresas. Una vez más, las revistas técnicas profesionales son consideradas de máximo impacto por sólo un $11 \%$ de los respondientes. En esta misma dirección, Allen (10) en su estudio encontró que la literatura constituía un $11 \%$ de las fuentes generadoras de ideas frente a un $42 \%$ proveniente de contactos directos entre colegas.

Conviene tener presente que el trabajo de Goldhar et al. (11) está basado en empresas punteras y con gran impacto en cuanto a sus procesos de innovación tecnológica. Los resultados esgrimidos más arriba parecen indicar, pues, que en la etapa de generación de ideas, después del primer estímulo proveniente de una necesidad externa, los innovadores confian fuertemente en su propio conocimiento y experiencia, las fuentes de información son fundamentalmente internas a la compañía y el intercambio verbal con los colegas es extremadamente importante. En suma, las fuentes y canales de información internos e informales juegan un papel clave en el proceso de estimulación de ideas innovadoras.

\section{Fase de resolución de problemas}

La tarea de resolución de problemas en un proceso de innovación tecnológica se suele considerar como diferenciado de alguna manera de lo que es en psicología el estudio de la conducta de resolución de problemas sobre todo porque, como afirman Johnson y Gibbons (12), en el proceso de innovación tecnológica no existe una solución correcta o mejor a largo plazo, sino que se trata de una relación dinámica entre problemas y soluciones. Los criterios establecidos para la consecución de los objetivos son una cuestión de juicio o de prioridades (8).

Johnson y Gibbons (12) estudiaron los modelos de uso de la información de equipos de tecnólogos en la etapa de resolución de problemas basándose para ello en una muestra constituida por los equipos de treinta compañias, todas ellas con gran impacto y éxito, y compararon sus resultados con los de estudios anteriores. En sus conclusiones, señalan que las fuentes de información externas representan un $34 \%$ del total de respuestas; las internas, un $30 \%$, y las fuentes personales, un $36 \%$. No obstante, aunque los resultados parecen discrepar con los de otros trabajos, hay que tener en cuenta que "fuentes externas" incluyen literatura impresa publicada fuera de la compañía, asi como contactos con colegas asimismo fuera de la firma. Las "fuentes internas", por el contrario, se refieren a contactos con colaboradores de dentro de la empresa y a literatura producida internamente. Las "fuentes personales» de información están relacionadas con la experiencia y la competencia profesional del innovador mismo. 
Este estudio intenta también analizar las fuentes de información específicas para cada una de las categorias señaladas en el párrafo anterior $\mathrm{y}$, contrariamente a otros trabajos, concluye que la incidencia total de literatura externa (un $29 \%$ ) alcanza una ratio casi tan alta como los contactos personales de todo tipo $(37 \%)$ en el hallazgo de soluciones a la resolución de problemas. Estos resultados contrastan fuertemente con los de Utterback (8) que reflejan el uso de literatura impresa en un $13 \%$ respecto al uso total de fuentes de información. Asimismo, los resultados alcanzados por Allen (10) son similares a los de Utterback.

A la vista de lo anterior, resulta dificil comparar datos estadísticos cuando las metodologías, fuentes y variables han sido definidas de modo diferente. De cualquier manera, es interesante subrayar el análisis que Johnson y Gibbons (12) hacen de la relación entre «contenido» y "fuente general». Sus resultados les llevan a concluir que en esta fase el innovador tecnológico utiliza primeramente su propio conocimiento basado en su experiencia y su competencia profesional, y cuando esto es insuficiente, utiliza selectivamente distintas fuentes para distintos tipos de información.

Asumiendo que la importancia de una fuente de información dada no está relacionada solamente con la frecuencia de su uso, en el trabajo de estos autores se trata de encontrar una correlación entre cada fuente de informacióny su impacto en la resolución de un problema. Con esta hipótesis de trabajo, nos encontramos con que la información obtenida fuera de la compañia parece contribuir significativamente más a la provisión de soluciones a problemas técnicos. Los contactos personales y materiales impresos tienen un impacto similar cuando son utilizados aisladamente pero el proceso de innovación es mucho más eficiente cuando ambos son usados conjuntamente.

Por lo que hasta aquí se desprende, se podría considerar esencial para un servicio de información que quiera maximizar el valor de la información que provee, el tratar de identificar los recursos que vayan a contribuir del modo más eficiente y efectivo a la resolución de los problemas tecnológicos.

\section{Patrones de comunicación en el proceso de innovación}

Hemos visto las fuentes y canales de información e, igualmente, el flujo de los diferentes tipos de información presentes en el proceso de innovación. Se ha señalado también la importancia de los contactos personales y de una buena comunicación para el éxito de la innovación tecnológica. Pues bien, algunos autores, entre ellos Robertson (13), refiriéndose a los resultados del Proyecto SAPPHO (Predictor de la Actividad Científica de Modelos con Origenes Heurísticos) señala que, si bien casi todas las compañías intentan tener «buenos contactos", lo que diferencia a los innovadores con éxito es que los últimos saben cómo hacer uso efectivo de estos contactos en su aplicación a proyectos específicos.

Según lo anterior, ¿el servicio de información debería tener en cuenta los patrones de comunicación de su clientela? ¿La biblioteca especial, la biblioteca de la empresa aumentaría el valor potencial de la información que provee si conociese los canales de comunicación de la compañía? ¿La red de comunicación informal es un medio no estructurado de flujo de información donde cada uno de los individuos se comunica con el resto tanto dentro como fuera de la firma? 
Se puede presumir que hay diferencias de comunicación entre individuos y que hay distintos tipos de contribución al contexto en el que la comunicación se desarrolla. De hecho, la investigación sobre la comunicación en el ámbito industrial resalta la existencia de individuos clave en el flujo informal de información técnica. Utterback (8) afirma que la comunicación fuera de la empresa tiende a concentrarse en unos pocos individuos que subsecuentemente actúan como recursos de información técnica dentro de la compañía. Estos comunicadores especiales son elegidos por el resto de los trabajadores por ser percibidos como poseedores de más y mejor información que otros y también por ser humanamente más accesibles (14).

Estos individuos clave se especializan en diversos tipos de fuentes. Los unos se caracterizan por ser ardientes lectores $y$, por tanto, por poner siempre a disposición el artículo más apropiado y reciente para una situación determinada; otros tienen los mejores contactos fuera de la empresa y proveen una continua puesta al día de lo que está sucediendo fuera de la compañía, etc... Allen (10) encontró distintos tipos de individuos clave en los departamentos de I + D. Este calificó como "guardianes de información tecnológica» a aquellos individuos que actuaban como consultores internos en aspectos técnicos, eran reconocida fuente de información externa o poseían numerosos contactos externos. Al mismo tiempo, estas personas publicaban más que los demás, poseian mayor número de patentes y su posición profesional dentro de la organización era intermedia.

Tushman (15), desde el marco del procesamiento de la información, sugiere que existen individuos que hacen de nexo de unión entre sus departamentos y el exterior, de tal manera que evitan a sus colegas, más orientados hacia lo doméstico o local, la turbulencia externa.

En cualquier caso, parece que la red de comunicación es una red estructurada y que afecta claramente a los resultados de los equipos de innovación tecnológica. Más aún, las conclusiones sugieren la existencia de individuos clave que actúan como empaquetadores o diseminadores de información y que son receptores y transmisores de fuentes de información tanto formales como informales. Las bibliotecas y los especialistas en información deberían ser sensibles a la existencia en la empresa de este tipo de personas $y$, específicamente, a sus necesidades de información, ya que de este modo se contribuiría más y mejor a la completa ejecución del proceso innovador.

\section{Valoración del servicio de información en la empresa}

Los resultados extraídos de la investigación revisada a lo largo de este trabajo muestran la variedad y complejidad del contexto informacional y de las necesidades de información cuando se pretende estudiar un proceso específico: fuentes formales de información coexisten con contactos personales; informes internos de la firma son, frecuentemente, sólo diseminados dentro de ella y, en algunos casos, materia de secreto; la información técnica parece dificil de documentar lo que promueve la comunicación verbal; existen individuos clave que multiplican la difusión de información y actúan como filtros y guardianes de ésta entre el mundo exterior y sus colegas. 
Todo lo anterior plantea, entre otras, la pregunta por el papel de la biblioteca servicio de información en la industria. Algunas de las cuestiones no contestadas son si la unidad de información es quien gestiona las fuentes formales de información tanto externas como internas, si la unidad de información trabaja codo con codo con los agentes de la innovación tecnológica, si aquélla está al corriente de la estructura de los patrones de comunicación, si las necesidades de información de cada usuario se analizan en función de la complejidad del medio y de su propio papel en éste, etc.

Nos vamos a valer de una encuesta que Matarazzo, Prusak y Gauthier (16) realizaron a los gerentes de 164 firmas americanas que representaban a los sectores comerciales e industriales que más contribuían al Producto Interior Bruto (PIB). El propósito del estudio no era otro que el de conocer el valor del servicio de información y del especialista en información desde el punto de vista gerencial, instancia de la que los anteriores dependen dentro de la empresa. Los resultados son útiles para nuestro estudio tanto por su actualidad como por el tema en sí.

Entre las conclusiones más pertinentes cabe señalar las siguientes:

a) Los bibliotecarios/especialistas en información y los gerentes evaluaban el ejercicio de su actividad de modo diferente. Mientras los primeros utilizaban metodologías biblioteconómicas estándar, los últimos utilizaban criterios subjetivos. El criterio argüido como más importante por éstos era la «calidad de la información" provista. No obstante, de acuerdo con Matarazzo et al. (16), muchos entrevistados respondían carecer de procedimientos de medida sobre el valor de la unidad de información.

A nuestro juicio, el que el estamento gerencial no disponga de criterios para evaluar el servicio de información es peligroso, quizá no tanto porque su existencia quede en entredicho, como por lo que puede incidir en su crecimiento y consideración dentro de la organización. Este tipo de servicios deberían estudiar medidas de evaluación válidas tanto para el servicio en sí como para los gestores.

b) Entre los tipos de información ofrecidos por el servicio, las búsquedas electrónicas fueron mencionadas como lo más valioso por un $80 \%$ de los respondientes. A la vista de la complejidad y variedad de fuentes y canales de información, la biblioteca/servicio de información debería, quizá, estudiar la contribución de este tipo de información al éxito empresarial. No obstante, en aras de un mayor incremento del potencial valor añadido a la información, la biblioteca debería ir más allá de la provisión de búsquedas electrónicas por petición previa.

Además, aunque todavía no se ha consolidado la consulta electrónica directa por parte del usuario final, las interfaces son, progresivamente, más fáciles de uso, homologadas, intuitivas, y accesibles para el usuario final. El bibliotecario/especialista en información no debería reducir su papel en la organización a ser un proveedor de «buenas búsquedas electrónicas», sino que acaso también debería estar más involucrado con su clientela y con el total sistema de flujos de información para poder, de este modo, responder más específicamente a las necesidades de información de los usuarios en un momento dado, en un punto dado del proceso.

c) Los resultados de esta encuesta ponen de manifiesto que el aparato gestor no sabe cómo el servicio de información contribuye a la marcha de la organización ni tampoco cómo se debería medir el valor de éste. Casi dos tercios de los 
entrevistados no sabían o no respondían a la pregunta de cuál de los servicios de la biblioteca añadía mayor valor a la empresa.

d) Los bibliotecarios/especialistas en información puntuaron alto en la mayor parte de las categorías seleccionadas. Tareas tales como desarrollar y difundir servicios efectivos y responder a las necesidades cambiantes de la firma fueron puntuadas alto por más del $70 \%$ de los entrevistados.

Más del $60 \%$ coincidían en señalar que los bibliotecarios contribuían en hacer productivo al personal. Algunos comentarios de los entrevistados subrayaban que el valor añadido provenía de la creación de servicios personalizados en lugar de servicios a la carta. Esto de alguna manera confirma lo que en otro lugar señalábamos: el papel del especialista provendria de desarrollar servicios de información más ajustados a su clientela y al medio en el que la información se utiliza.

Los profesionales de la información son vistos como organizadores y diseminadores de información pero raramente son analistas de datos. Los encuestadores sugieren que ello incrementaría el valor del servicio de información. En nuestra opinión no está claro que esta preparación aumentase automáticamente el valor del servicio, pero sí se podría sugerir que, siendo el bibliotecario especialista en un área o disciplina dada, no sería un experto en información sino un experto doble $y$, en este sentido, podría más fácilmente ser un guardián de la información, en el sentido en que antes se definía este concepto, para las fuentes formales de esa determinada disciplina.

e) Otra conclusión seria que se deriva de este estudio es lo poco que los servicios de información dicen en la política de información de la empresa. De acuerdo con los resultados, muy pocos entrevistados pudieron fijar la función exacta de la biblioteca en la estructura de información de la firma.

No es ciertamente halagüeño que en la era de la "sociedad de la información», la biblioteca/unidad de información tenga un papel tan indefinido como para no ser considerada parte activa y de pleno derecho en la estructura del sistema de información de la organización.

f) Por último, el estudio resalta que por parte de los entrevistados (no olvidemos que hablamos de una sociedad donde la biblioteca pública está enraizada en la vida cotidiana) existe un profundo afecto por las bibliotecas y por los especialistas en información, a menudo basado en una percepción intuitiva de que el servicio es valioso y de que merece la pena mantenerlo.

No obstante, los servicios y especialistas en información, a mi entender, no tenemos mucho que ganar en esta perspectiva. Yendo un poco más lejos, deberíamos tratar de eliminar este concepto e intentar redefinir los objetivos y el valor de la información en la industria de tal manera que ambos, servicio y especialista, fueran valorados en sí mismos por la contribución que hacen al desarrollo de la corporación.

\section{A modo de conclusiones}

En este trabajo empezábamos preguntándonos por la información en la industria para considerar a continuación algunas aproximaciones al concepto de "valor de la información» tomando al usuario y su entorno como variables 
esenciales. Se han manifestado también las dificultades y limitaciones para asignar tal valor a una pieza de información cuando es entendida como un proceso dinámico. Posteriormente, se han analizado y comentado los estudios sobre flujos de información y transferencia de información en la innovación tecnológica en un intento de reflejar en un contexto particular las características de uso, del usuario y del medio, variables todas ellas intervinientes en un enfoque de valor de la información como el que aquí se ha presentado.

Ha quedado de manifiesto la dificultad, cuando no imposibilidad, de asignar valor a una pieza de información al menos en contextos donde ésta fluye a través de múltiples vías como en el caso de las unidades de I + D estudiadas: variedad de fuentes de información, conocimiento individual y experiencia profesional parecen estar interactuando permanentemente y es solamente en función de esa interacción como el valor añadido real puede ser tratado y analizado.

No obstante, a la vista de lo expuesto, cabe decir que la unidad de información puede contribuir y aumentar el valor añadido potencial de la información tomando en cuenta las particularidades del usuario y su contexto. Como decía Yates (17), a la unidad de información «le corresponde una parte en la conversión de las ideas en acciones, pero sólo una parte" (has a part in turning ideas into actions, but only a part). Es por ello incluso más importante conocer a fondo las características y necesidades del usuario, los canales de comunicación de la clientela atendida por la biblioteca, los tipos y fuentes de información más apropiados para cada fase de un proceso o contexto determinado.

Desarrollar servicios efectivos quiere decir, en mi opinión, contribuir a optimizar, no cuantitativa sino cualitativamente, el uso de la información, aumentar el valor añadido potencial de la información, y como los gerentes del estudio de Matarazzo recalcaban, ello conlleva el desarrollo de servicios más a la medida. Cabe preguntarse hasta qué punto el especialista en información, al menos en ámbitos industriales y en los de disciplinas específicas, debería tener también una formación académica acorde con el área específica. Quizás ello contribuiría a que fuese percibido como un interpar o colaborador más en la estructura de los flujos de información y, tal vez, a tener voz en las directrices del sistema de información de la organización.

Harian falta más estudios para conocer las complejas estructuras de transferencia de información no sólo en ámbitos industriales sino también en marketing, en los procesos de toma de decisiones en la empresa, en medios académicos y, en general, en aquellos ámbitos atendidos por los servicios de información. Aquí se ha descrito un medio controlado y bastante bien conocido. Bastante más complejo de analizar serían, por su diversidad, los contextos de uso de información del «gran público». Sin embargo, parece esencial tratar de conocer las necesidades de información del usuario y el contexto donde esa información es aplicada, sobre todo si nuestro objetivo es lograr el máximo valor potencial de la información que proveemos al usuario.

Parafraseando a Yates (17) yo también diría que «la unidad de información nunca encontrará su lugar verdadero hasta que no se pregunte a sí misma qué es lo que debemos hacer para asegurar la acción como resultado de la información que proveemos" (the information unit will never find its true place until it asks itself what should we do to ensure action as a result of information we provide). Visto 
desde otra perspectiva, ¿qué es lo que deberiamos hacer para aumentar el valor añadido potencial de la información que proveemos? No parece haber una respuesta que dé solución a ello, pero, quizás, conocer las necesidades del usuario, las características del medio en el que aplica la información y, en algunos casos, tener una información en la disciplina de la comunidad a la que atendemos sean vías para conseguirlo.

\section{Bibliografia}

1. RUIZ GONZALEZ, B.; GONZALEZ MOLINA, M. T. (eds.). Catálogo de servicios españoles de información electrónica ASCII. 6. ${ }^{\mathrm{a}}$ ed. Madrid: FUINCA, 1991.

2. CRONIN, B.; GUDIM, M. Information and Productivity: A Review of Research. International Journal of Information Management, 1986, 6 (2), 85-101.

3. TAYLOR, R. Value-Added Processes in the Information Life Cycle. Journal of the American Society for Information Science, 1982, 33 (5), 341-346.

4. MARSCHAK, J. Economic Information: Decision and Pediction. Selected Essays. Vol. 2. Hingham, MA: Reidel, 1974.

5. OZOG, S. On the value of information. Journal of the American Society for Information Science, 1979, 30, 310-315.

6. KING, D. W. et al.. The Value of the Energy Database. Rockville, MD: King Research Inc., 1982.

7. REPO, A. J. The dual approach to the value of information: an appraisal of use and exchange values. Information Processing \& Management, 1986, 22 (5), 373-383.

8. UTTERBACK, J. M. The Process of Technological Innovation Within the Firm. Academy of Management Journal, marzo 1971, 14, 75-88.

9. BATTELLE COLUMBUS LABORATORIES. Science, Technology and Innovation. Washington, D. C.: National Science Foundation, febrero 1973.

10. ALLEN, T. J. Managing the flow of technology: technology transfer and the dissemination of technological information within the R\&D organization. Cambridge, MA: MIT Press, 1977.

11. GOLDHAR, J. D.; BRAGAW, L. K.; SCHWARTZ, J. J. Information Flows, Management Styles and Technological Innovation. IEEE Transactions on Engineering Management, 1976, EM-23 (1), 51-56.

12. JOHNSTON, R.; GIBBONS, M. Characteristics of Information Usage in Technical Innovation. IEEE Transactions on Engineering Management, 1975, EM-22 (1), 27-34.

13. ROBERTSON, A. Information flow and industrial innovation. ASUB Proceedings, 1973, 25 (4), 130-139.

14. HOLLAND, W. E. The Special Communicator and His Behavior in Research Organizations: A Key to the Management of Informal Technical Information Flow. IEEE Transactions on Professional Communication, 1974, PC-17 (3/4), 48-53.

15. TUSHMAN, M. L. Impacts of Perceived Environmental Variability on Patterns of Work Related Communication. Academy of Management Journal, 1979, 22 (3), 482-500.

16. MATARAZZO, J. M.; PRUSAK, L.; GAUTHIER, M. Valuing Corporate Libraries: A Survey of Senior Managers. Washington, DC: Special Libraries Association, 1990.

17. YATES, B. The place of the innovation service within the organizational structure. ASUB Proceedings, 1973, 25 (11), 430-444. 